\title{
Rapidly progressive Aspergillus meningitis successfully treated with voriconazole and corticosteroids
}

\author{
Akiyuki Hiraga $^{1}$ (D) Reiji Aoki $^{1} \cdot$ Keigo Nakamura $^{1} \cdot$ Satoshi Kuwabara $^{2}$
}

Received: 2 September 2017 / Accepted: 3 October 2017 /Published online: 7 October 2017

(C) Springer-Verlag Italia S.r.1. 2017

\section{Dear Editors,}

Central nervous system (CNS) aspergillosis is a frequently fatal infection that mainly affects immunosuppressed patients. Mortality rates in patients receiving voriconazole (VRZ) treatment remain unacceptably high, and more efficacious therapies are needed [1]. Unlike bacterial or tuberculosis meningitis, corticosteroid treatment for CNS aspergillosis has not yet been established. We report the case of a young immunocompetent woman with acute Aspergillus meningitis presenting with rapidly progressive respiratory failure and successfully treated using VRZ and a corticosteroid.

Case report A 21-year-old immunocompetent woman with no medical history developed a frontal headache, followed by appetite loss and fever. Abdominal and chest computed tomography (CT) performed at a local hospital showed no abnormalities. She was referred to our hospital 8 days after the onset of headache. On admission, her body temperature was $38.4{ }^{\circ} \mathrm{C}$, and she was alert and cooperative. She had neck stiffness. No cranial nerve palsies, motor paresis, or sensory disturbances were observed. Enzyme-linked immunosorbent assay was negative for human immunodeficiency virus. Her CD4 cell count was $470 / \mu \mathrm{L}$, which was $39.9 \%$ of the total lymphocyte count. Cerebrospinal fluid (CSF) analysis revealed a white blood cell count of $96 / \mathrm{mm}^{3}$ (12\% neutrophils and $88 \%$ lymphocytes) and a glucose level of $35 \mathrm{mg} / \mathrm{dL}$

Akiyuki Hiraga

hiragaa@yahoo.co.jp

1 Department of Neurology, Chiba Rosai Hospital, 2-16 Tatsumidai-Higashi, Ichihara-shi, Chiba 290-0003, Japan

2 Department of Neurology, Graduate School of Medicine, Chiba University, Chiba, Japan (simultaneous blood sugar, $102 \mathrm{mg} / \mathrm{dL}$ ). CSF bacterial cultures were negative for pathogens and CSF cryptococcal antigen. The results of polymerase chain reaction (PCR) analysis of CSF were negative for tuberculosis. CSF viral titres were negative. Brain CT showed no abnormalities. Although the CSF glucose level was mildly lower than normal, the patient's clinical history of acute symptoms, physical examination findings, and elevated CSF lymphocyte levels were consistent with a diagnosis of aseptic meningitis; therefore, we chose conservative therapy. On day 2 of admission, her level of consciousness deteriorated that was incompatible with the diagnosis of aseptic meningitis. Brain magnetic resonance imaging (MRI) including the paranasal area showed no abnormalities. We suspected viral encephalomeningitis and administered intravenous acyclovir. However, her symptoms did not improve, and she became comatose and developed respiratory failure on the following day. She was transferred to the intensive care unit and was intubated and placed on a ventilator. Thereafter, spontaneous respiration stopped, and brain CT showed bilateral cerebral oedema (Fig. 1). Intravenous methylprednisolone was administered ( $1 \mathrm{~g} /$ day for 3 days), followed by prednisolone via a nasogastric tube. The disturbance of consciousness, respiratory failure, and brain oedema improved after corticosteroid treatment (Fig. 1). Serum 1,3- $\beta$ D-glucan (BG) values obtained using the Fungitec $G$ Test MKII (Nissui Pharmaceutical, Tokyo, Japan) were elevated to $230 \mathrm{pg} / \mathrm{mL}$ (normal, $<20 \mathrm{pg} / \mathrm{mL}$ ), and a test for CSF Aspergillus galactomannan (GM) antigen performed using enzyme-linked immunosorbent assay (Plateria Aspergillus, Bio-Rad) showed an optical density of 0.6 (cut-off, $<0.5$ ). PCR analysis of CSF was negative for Aspergillus, and CSF was negative for Candida antigen. Based on these findings, a diagnosis of acute Aspergillus meningitis was made. VRZ was intravenously administered beginning on day 10 of admission. The neurologic symptoms resolved and treatment 
Fig. 1 Serial brain computed tomography at (a) admission, (b) 3 days after admission (showing bilateral brain oedema) and (c) 9 days after admission (showing amelioration of brain oedema after corticosteroid therapy)

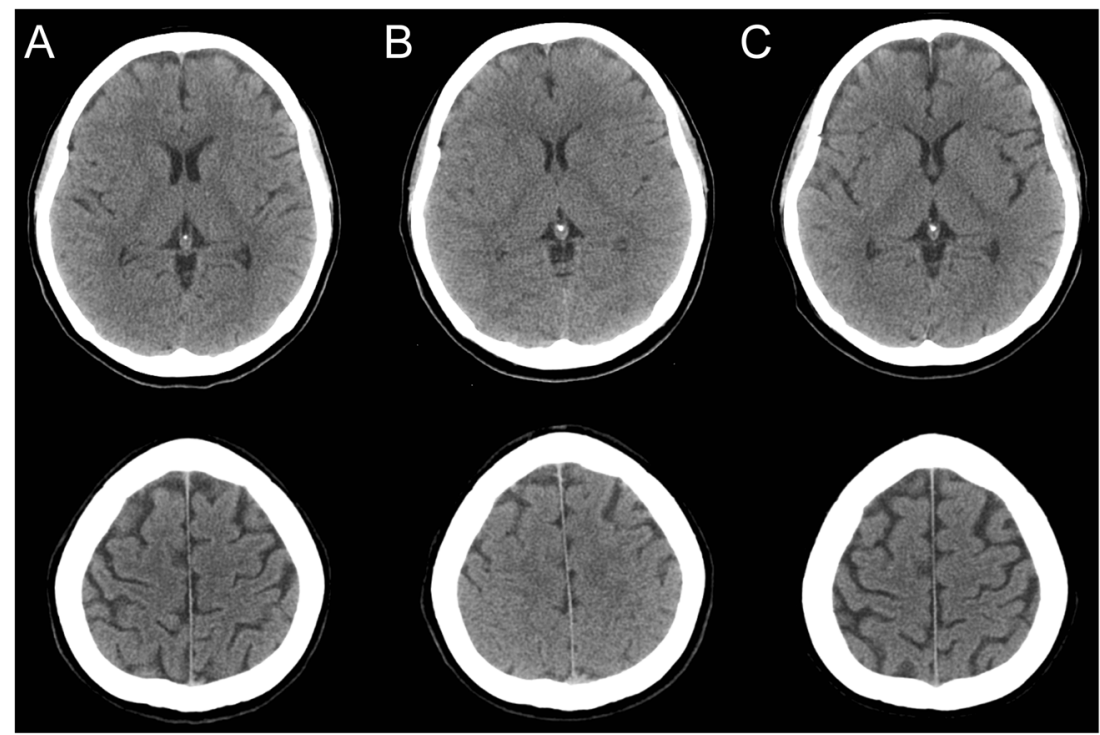

continued with oral VRZ and oral prednisolone. Blood cultures on both days 5 and 13 after admission were negative, and an ultracardiogram detected no vegetation. Post-contrast brain MRI showed no abnormally enhanced lesions. Whole-spine MRI showed no abnormalities and no sign of abscess or discitis. Her urine and sputum cultures were negative for Aspergillus. These findings indicated that the focus of infection of aspergillosis was uncertain. Aspergillus GM antigen in CSF was 0.0 on day 17 and persisted at 0.0 on days 28 and 38 after admission and serum BG values levels normalised. The patient showed good clinical recovery without residual signs or symptoms, and VRZ was discontinued 6 months after initiation. Relapse had not occurred at 12 months after onset.

Our patient was diagnosed with rapidly progressing acute Aspergillus meningitis with respiratory failure. In managing this patient, we identified two key clinical issues. First, young immunocompetent patients may present acute meningitis mimicking aseptic meningitis, without a focus of aspergillosis, such as the lungs or paranasal sinuses, and testing for CSF Aspergillus GM antigen is diagnostically useful in such cases. Second, corticosteroid treatment combined with VRZ was effective for our patient with CNS aspergillosis.

Aspergillus meningitis is an infrequently encountered clinical entity, and its diagnosis is extremely challenging. Its diagnosis during life is observed only in $55.9 \%$ of patients, and the overall mortality rate is $72.1 \%$ [2]. Potential sites of primary infection in CNS aspergillosis include the paranasal sinuses, lungs, and other sites; however, similar to our patient, $22 \%$ of patients have no apparent primary site of infection [1]. Brain biopsy or lowsignal-intensity T2-weighted MRI is a diagnostic tool for fungal infection; however, it is difficult to diagnose Aspergillus meningitis when brain abscesses and primal organ involvement are absent in immunocompetent patients. CSF cultures are usually negative, and the cell count and glucose and protein concentrations are nonspecific in CNS aspergillosis. Moreover, the results of a CSF PCR analysis may be negative for the fungal agent in CNS aspergillosis, because the number of fungal cells present in a CSF specimen obtained via lumbar puncture is extremely low. Therefore, the detection of the CSF Aspergillus GM antigen might be the earliest indicator of the condition and be useful for monitoring treatment response [3]. Indeed, previous reports comparing CSF PCR analysis and CSF antigen assays for CNS aspergillosis showed that in some patients, assays are positive for Aspergillus GM antigen, but PCR results are negative [3].

In our patient, corticosteroid treatment dramatically reduced brain oedema and improved clinical symptoms. In the Infectious Disease Society of America treatment guidelines for CNS aspergillosis, corticosteroids are considered to be deleterious and should be avoided [4]. However, we have previously reported the successful treatment of CNS aspergillosis in an immunocompetent patient with VRZ and corticosteroids [5]. Unlike the present patient, the previous patient was characterised by gadolinium-enhanced extramedullary lesions (presenting aspergilloma) with intramedullary pontine and cerebellar T2 hyperintense lesions. The extramedullary lesions were ameliorated by VRZ, and the intramedullary lesions, which worsened during treatment with VRZ alone, were resolved by corticosteroid treatment. The cases of these two patients indicate that both direct ongoing infectious damage and secondary inflammation are present in CNS aspergillosis. These cases also raise the possibility that advanced treatment of secondary inflammation with corticosteroids combined with anti-fungal drugs is effective in this condition. Immunosuppressed patients with CNS aspergillosis might be unable to tolerate corticosteroid treatment; however, patients 
with CNS aspergillosis with secondary inflammation may be candidates for combined corticosteroid and VRZ therapy if there are no indications of immunosuppression.

The limitation of this case report is that we did not perform fungal culture, and GM antigen level in CSF is lower than that reported in a previous systematic review (range, 2.2-578) [2], suggesting that other causes of meningoencephalitis cannot be completely excluded. If the patient had viral or autoimmune meninoencephalitis, the patient probably improved by corticosteroid. Actually, some virus could not be identified by usual testing. Although the appropriate cutoff value of GM antigen level in CSF has not been established till date, the decline in GM antigen level in CSF, which corresponded with the clinical response to treatment with VRZ in the present case, indicates that the diagnosis of Aspergillus meningitis might be appropriate and was managed successfully. Factors that cause false-positive results of BG or Aspergillus GM antigen, such as albumin, intravenous immune globulin, gauze packing, cellulose depth filters, beta-lactam antimicrobials, or soybean proteins were excluded in this case. However, cross-reactivity of other fungi was not completely excluded.

In conclusion, combination therapy with VRZ and corticosteroid may be effective for the treatment of certain patients with CNS aspergillosis. The diagnosis of acute Aspergillus meningitis without a primary focus, such as the paranasal sinuses or lungs, in immunocompetent patient is still challenging. Testing for CSF GM antigen is probably a useful diagnostic tool.

\section{Compliance with ethical standards}

Conflict of interest The authors declare that they have no competing interests.

\section{References}

1. Kourkoumpetis TK, Desalermos A, Muhammed M, Mylonakis E (2012) Central nervous system aspergillosis: a series of 14 cases from a general hospital and review of 123 cases from the literature. Medicine (Baltimore) 91:328-336

2. Antinori S, Corbellino M, Meroni L et al (2013) Aspergillus meningitis: a rare clinical manifestation of central nervous system aspergillosis. Case report and review of 92 cases. J Inf Secur 66:218-238

3. Verweij PE, Brinkman K, Kremer HP, Kullberg BJ, Meis JF (1999) Aspergillus meningitis: diagnosis by non-culture-based microbiological methods and management. J Clin Microbiol 37:1186-1189

4. Patterson TF, Thompson GR III, Denning DW et al (2016) Practice guidelines for the diagnosis and management of aspergillosis: 2016 update by the Infectious Diseases Society of America. Clin Infect Dis 63:e1-e60

5. Hiraga A, Uzawa A, Shibuya M, Numata T, Sunami S, Kamitsukasa I (2009) Neuroaspergillosis in an immunocompetent patient successfully treated with voriconazole and a corticosteroid. Intern Med 48: $1225-1229$ 